\title{
Commentary: Concerns Regarding FDA Guidance on Dissolution Testing
}

Alger Salt

Sal Mea Consulting, Durham, NC, USA

e-mail: algersalt@gmail.com

C oncerns have been raised regarding the recent US Food and Drug Administration (FDA) guidance on dissolution testing. I share these apprehensions here and welcome any additional information or comments you may have on this topic.

In 1997, the FDA released an industry guidance entitled Dissolution Testing of Immediate Release Solid Oral Dosage Forms (1). At that time, the established biopharmaceutical classification system (BCS) categorized pharmaceutical actives in four discrete classes according to solubility and drug absorption or permeability. Highly soluble drugs were listed as Class 1 and Class 3. In 2015, nearly 18 years later, a draft guidance Dissolution Testing and Specification Criteria for Immediate-Release Solid Oral Dosage Forms Containing Biopharmaceutics Classification System Class 1 and 3 Drugs was issued for public comment (2). However, a subsequent draft revision reflecting the comments received was never issued. The current guidance Dissolution Testing and Acceptance Criteria for Immediate-Release Solid Oral Dosage Form Drug Products Containing High Solubility Drug Substances, Guidance for Industry was issued in August 2018 (3). Thus, the official guidance was released 3 years after the draft guidance without any opportunity for the external community to review or comment.

The official guidance reflected changes that were never presented in the draft, thereby creating a regulatory gap or mismatch. One example is that Brazil (and perhaps some other countries) reacted to the draft guidance and moved forward with policy based on this (4). It is alarming that some critical issues were omitted and/or changed between the draft and the official version. Specifically:

- The option to substitute a disintegration test for rapidly dissolving formulations is not included in this guidance. Disintegration is an alternate test for assessing the performance of a formulation and in some cases can be more discriminating and provide better information than a dissolution test;

- The acceptance criteria changed from a value of $Q=$ $85 \%$ at 15 minutes to $80 \%$ at $30 \mathrm{~min}$. A reason for this change was not provided; and
- The previous guidance suggested the use of $0.01 \mathrm{~N}$ $\mathrm{HCl}$ as an alternate medium to $0.1 \mathrm{~N} \mathrm{HCl}$. This does not appear in the new guidance. It had been proposed that the alternate medium was more biorelevant and more environmentally friendly than $0.1 \mathrm{~N} \mathrm{HCl}$.

The public was not given sufficient opportunity to review and rebut some of the changes between the original guidance and the current guidance that was released in August 2018. FDA guidance documents are important and very useful to the pharmaceutical industry, especially to those involved in the development of formulations, processes, and testing procedures for new drug applications. The FDA should consider more allowance for public input prior to the release of future guidance documents.

\section{CONFLICT OF INTEREST}

The author disclosed no conflict of interest related to this article.

\section{REFERENCES}

1. Dissolution Testing of Immediate Release Solid Oral Dosage Forms Guidance for Industry; U.S. Department of Health and Human Services, Food and Drug Administration, Center for Drug Evaluation and Research (CDER), U.S. Government Printing Office: Washington, DC, 1997.

2. Dissolution Testing and Specification Criteria for ImmediateRelease Solid Oral Dosage Forms Containing Biopharmaceutics Classification System Class 1 and 3 Drugs, Draft Guidance for Industry; U.S. Department of Health and Human Services, Food and Drug Administration, Center for Drug Evaluation and Research (CDER), U.S. Government Printing Office: Washington, DC, 2015.

3. Dissolution Testing and Acceptance Criteria for ImmediateRelease Solid Oral Dosage Form Drug Products Containing High Solubility Drug Substances, Draft Guidance for Industry; U.S. Department of Health and Human Services, Food and Drug Administration, Center for Drug Evaluation and Research (CDER), U.S. Government Printing Office: Washington, DC, August 2018.

4. Dissolution Guide Applicable to Generic, New and Simlar Medicines, Agência Nacional de Vigilância Sanitária: Brazil, May 2018. 